\title{
A REVIEW ON ENERGY EFFICIENT CLUSTERING ROUTING PROTOCOL IN WIRELESS SENSOR NETWORK
}

\author{
Rahul Goyal \\ Student, Department of Computer Engineering, Punjabi University Patiala, Punjab, India
}

\begin{abstract}
Wireless Sensor Network depends on nodes have limited energy, memory, computational power, range and it is important to increase energy efficiency by saving the battery power so as to extend the life time of the given WSN deployment. In WSN, data is measured by nodes and same is send to Base Station at regular interval. Different protocols are used for energy consumption, in wireless sensor network. In this paper for energy consumption in wireless sensor network we study about LEACH protocol, EEUC protocol and zone-divided and energy-balanced clustering routing protocol (ZECR) which divides the area into several zones according to the distance and uses the clustering method among these zones. The key idea of these protocols is that within a cluster there is a "cluster-head" which receive the data from remaining nodes and such data is sent to base station.
\end{abstract}

Keywords-Wireless Sensor Networks, Sensor Node, Routing, LEACH, Leach-FL, EEUC, ZECR,EEZECR.

\section{INTRODUCTION}

Wireless Sensor Networks (WSN's) are being used in physical phenomena like surveillance, industrial monitoring, traffic monitoring, habitat monitoring, cropping monitoring, crowd counting etc. which calls for monitoring before taking an appropriate action.WSN typically a geographical area in which hundreds or thousands of sensor nodes that are deployed to sense events. Many academic attentions have received by Wireless sensor networks (WSN) and this technology is considered with huge influence in the current century [1].

The growing use and recent development in these networks is making engineers to evolve innovative and efficient ideas in this field. In recent years, a lot of research has been proposed in data compression, data routing, and in-network aggregation.

In wireless sensor network, sensor nodes used are limited in power, memory, capacities and computational power. So, for long time working we need to save energy and balancing the energy consumption [2]. It is important role of routing protocol in wireless sensor network to save battery life. The energy is mainly consumes in receiving and sending data. But a significant amount of energy is wasted with regard to data collision, data overhearing, idle listing, interference, control packet overhead [3].

Wireless sensor network, spread over a specific area where we want to look after at the changes going on there. Within that area we use wireless medium by which sensor nodes can communicate with each other. Wireless medium used by many ways like, radio frequencies, infrared, satellite and any other medium. The sensor nodes plays the role of ad hoc network as these nodes deployed in random fashion for communicate among themselves. These nodes communicate either by direct link or by multi- hoping. The concept of multi-hoping is further modified as by making clusters. In clusters, there is a cluster-head which receive the data from remaining nodes and such data is sent to base station. In this paper I am going to discuss these protocols: LEACH protocol, LEACH-FL, EEUC protocol and ZECR protocol, EEZECR protocol. Clustering algorithms can achieve higher energy efficiency as compared to non clustering algorithm.

\section{PROBLEMS IN WSN}

There are various problems in Wireless sensor network. Coverage problem, which reflects how well a sensor network is monitored or tracked by sensors. Position estimation problem, which relates to the distance measures between sensor positions. Rest of all these problems one of the fundamental issue is energy conservation of sensors. In Wireless sensor network, most of the energy is consumed in transmission and receiving of data as compared to sensing and processing of data [3].

\section{ROUTING PROTOCOLS IN WSN}

In recent years, many clustering routing protocols are used in wireless sensor network. In our study the main focus is on LEACH, EEUC and ZECR routing protocols.

\subsection{LEACH (Low-Energy Adaptive Clustering}

\section{Hierarchy)}

It is hierarchical routing algorithm based on clustering. In each round every node has the probability to get selected as cluster head [4]. It proposes the concept of round for the implementation of periodicity. It involves two phases in every round: cluster set-up phase and steady data transmission phase [5]. In sensor network algorithm is used to divide into clusters. So the communication loads are shared and the energy consumption of every part of the 
network is balanced. The network topology shaped by LEACH is shown:

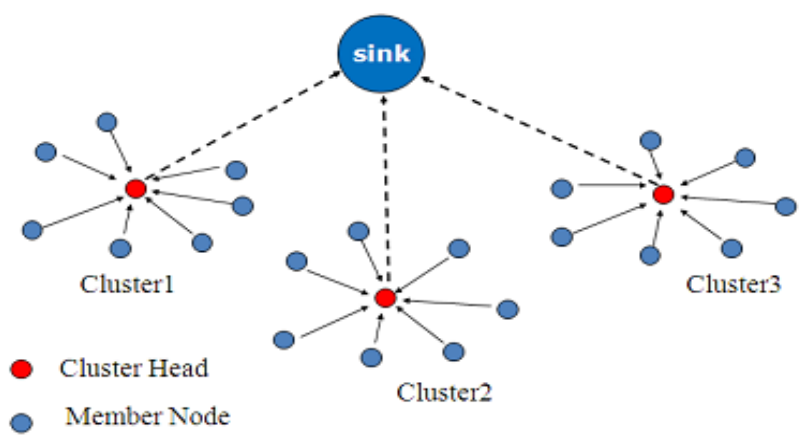

Fig.1. Leach Protocol [6]

\subsection{LEACH-FL (Low-Energy Adaptive Clustering}

\section{Hierarchy using Fuzzy Logic)}

This method, improves the LEACH protocol by using fuzzy logic on LEACH protocol. Selection of cluster head is based on three variables -battery level of node, node density and distance from base station. In this system, we assume that all the nodes can get their coordinates in WSN. LEACH-FL has three parts-four fuzzification, functions, an inference engine (include 27 rules) and defuzzification module [5].

\subsection{EEUC Protocol (Energy Efficient Unequal}

\section{Clustering)}

The purpose of using EEUC Protocol is to solve the hot spots problem that is caused due to the inter-cluster multi hop routing. It can avoid the energy hole and balance the energy consumption of the whole network [7].

The clustering strategy of EEUC is: The cluster radius is set according to the distance between node and Base station, so larger cluster are formed in the region far from base station. EECU network is as

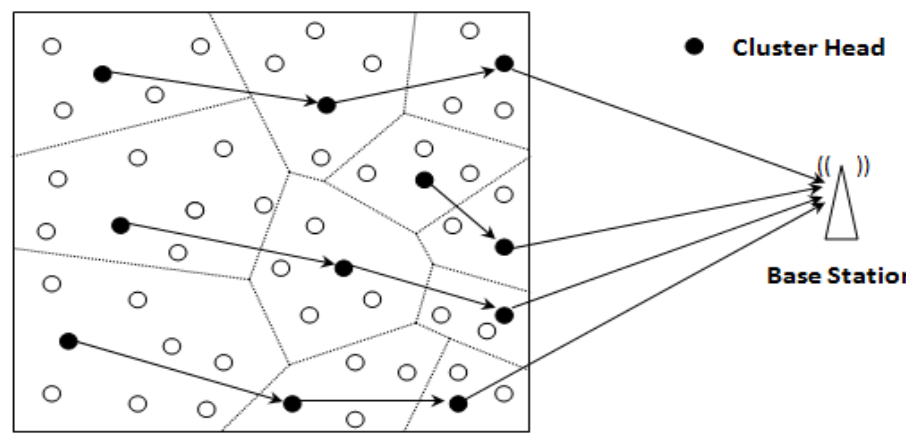

Fig 2 Overview of the unequal clustering [1]

\subsection{ZECR (Zone-Divided and Energy -Balanced}

\section{Clustering Routing)}

ZECR protocol is a very efficient protocol for WSN and adapts to the energy heterogeneous network. It can balance the energy consumption of the network and prolong the network life time obviously. In this section the details of ZECR protocol will be illustrated by the five parts in sequence: Zone division, Size of cluster radius, Cluster setup phase, Inter-cluster multi-hop routing phase and Data transmission phase [1].

\subsection{EEZECR (Energy Efficient Zone Divided and Energy Balanced Clustering Routing Protocol)}

In this method, concept of two cluster heads is introduced that make ZECR protocol more energy efficient. In the network, different zones are divided by using zone divisional approach. Two cluster heads used are - Assistant Cluster Head (ACH) and Main Cluster Head (MCH). ACH will collect the data from all the nodes in cluster and from that appropriate data is send to $\mathrm{MCH}$. Then $\mathrm{MCH}$ send that data to $\mathrm{MCH}$ of neighbor cluster that is nearer to base station. In this method, ACH reduced the load from $\mathrm{MCH}$ and this will make multi-hop transmission more energy efficient [8].

\section{RELATED WORK}

Hwa Young Lim et al [9] for solving the problem of LEACH a routing protocol called Maximum Energy routing protocol based on Strong Head (MESH) is proposed. In this protocol, the data collected by cluster head is send to Base station by node known as "Strong Head". Simulation results show that energy consumption and lifetime of the network improves.

Bilal Abu Bakr, Leszek Lilie [10] they propose a protocol which provides an optimal energy- saving spare management, including spare selection, known as LEACHSM Protocol. LACHSM protocol is modified form of LEACH Protocol in which Spare selection phase is added in LEACH. Quantitative comparison of energy consumption and WSN lifetime for both protocols is presented in this paper.

Chi-Tsun Cheng et al [11] proposed a delay-aware data collection network for WSN. By this network structure, delays in the data collection processes of WSN, is minimized. To construct the network structure for centralized and decentralized approach, two network formation algorithms are designed. By computer simulation performance of network structure is evaluated. Simulation results show that the proposed network structure is able to shorten the delays in the data collection process significantly as compared to other network structure.

Maciej Nikodem and Bartosz Wojciechowski [12] focus on the theoretical aspects of clustering to improve the lifetime of network, in WSN. By taking into account capabilities of real-life nodes in particular application and when compared to non-clustering networks they investigate whether clustering itself can improve network lifetime. Results show that some additional techniques and means are required with clustering to improve network lifetime. 
Yan Shen, Hui Ju [13] new energy-saving task assignment method is proposed in this paper. Based on the entropy theory according to the nodes status, such as computing resources, the residual energy and the number of neighbor, a cost function is described in this method. To optimize dynamic test assignment particle swarm optimization algorithm is used. Due to the changes in sensor network the tasks can be adjusted dynamically. Simulation results show that, lifetime of network is improved and energy consumption and execution time decreases.

F.J.Ateroetal [14] proposed a optimized power saving clustering algorithm, which builds inter-cluster and intracluster hierarchical trees, known as Hierarchical Adaptive and Reliable Routing (HARP). In both homogenous and heterogeneous network this architecture is used. By means of the addition of a recovery slot in the scheduling scheme, efficient link fault tolerance is provided by HARP and it also supports node mobility management. For newly deployed nodes, the same process function as a joining mechanism. This architecture provides bounded-time data transmission and highly adaptive for specific application requirements. With data gathering protocol, a new cluster head election formula is proposed. In a network, this protocol optimizes and balances the energy consumption.

Rongxin Li et al [15] present several routing protocols that increase the lifetime of network. Simulation results by NS2 show that the network lifetime of proposed routing protocol is better than other routing protocols.

Jacques M. Bahi et al [16] introduce an efficient distributed failure-aware strategy using probabilistic Weibull distribution to resist frequenting and unexpected failsilent/fail-stop node failures. To show the usefulness of the proposed solution, they provide a comprehensive set of experimental results.

Nagarajan.M. Dr. S. Karthikeyan [17] proposed an algorithm for WSN which increase the lifetime of sensor nodes. Only few sensors are in active state in the covered regions and the remaining are in ideal. All nodes change their status from ideal to active and active to ideal after time period. Active nodes are in active state or not are checked by nodes that are ideal for short period of time and these ideal nodes get active if any failure in the region. this process is periodic, when active nodes start sense the data the energy of ideal nodes is saved and it will used when it gets active. The performance of proposed algorithm is six times better than that of existing algorithms and it is close to optimal enhancement in the network.

Young Sang Yun et al [18] proposed that when there is a mobile sink and the underlying application can tolerate some amount of delay in delivering the data to the sink, an algorithm for WSN that minimizes the lifetime of network. The algorithm is distributed and in addition, mostly uses local information. By parallel or distributed execution this algorithm can be implemented. The overhead of message passing is low. The algorithm can be run directly by sensor node and sink, if we embed the algorithm into network protocol.

Jia Xu et al., [19] proposed a revised cluster routing algorithm to enhance the hierarchical routing protocol LEACH known as E-LEACH. The way of the selection of the cluster heads in the LEACH is random and the round time is fixed for selection. In the E-LEACH algorithm, we consider the remnant power of the sensor nodes in order to balance network loads and changes the round time depends on the optimal cluster size. The simulation results show that lifetime of network is increased by $40 \%$ when compared with LEACH algorithm

\section{CONCLUSIONS}

In Wireless sensor network, energy is an important issue. So designing energy efficient protocol is very important. In this paper, different energy efficient routing protocols that are based on clustering mechanism have been discussed. The EEZECR protocol can be used to improve the energy at great extent. So in future we will propose a new algorithm by using clustering technique to improve the results further.

\section{REFERENCES}

[1] Zou Yun, Jia Xibei et al "Zone-Divided And EnergyBalanced Clustering Routing Protocol For Wireless Sensor Networks"4th IEEE International Conference on Broadband Network and Multimedia Technology (IC-BNMT), pp. 112 - 117, 28-30 Oct. 2011.

[2] Kodali R.K., Sarma N "Energy Efficient Routing Protocols for WSN's" international conference on computer communication and information, pp 1-4, 46 jan, 2013.

[3] Rahman A., Anwar S. et al "A Survey on Energy Efficient Routing Techniques in Wireless Sensor Network" international conference on Advanced Communication Technology, pp 200-206,27-30 jan,2013.

[4] Naik R.K.,Bhardwaj H. et al "Residual Energy Analysis of DEC and Delivery Ratio Analysis of EAOMDV protocol for Mobile Sensor Networks" international conference on Communication and Signal Processing, pp 509-513, 3-5 april,2013.

[5] Ran Ge, Zhang Huazhong et al "Improving on LEACH Protocol of Wireless Sensor Networks Using Fuzzy Logic" Journal of Information \& Computational Science 7: 3, pp. 767-775, March 2010.

[6] Ben Alla S., Mohsen A. et al "Hierarchical adaptive balanced energy efficient routing protocol (HABRP) for heterogeneous wireless sensor networks" international conference on Multimedia Computing And Systems, pp 5-6, 7-9 april 2011.

[7] Ye Mao, Wu Jie et al "An Energy-Efficient Unequal Clustering Mechanism for Wireless Sensor Networks" international conference on Mobile Ad hoc and Sensor System Conference, pp 604, 7 nov 2005. 
[8] Verma S., Sharma K. "Energy Efficient Zone Divided and Energy Balanced Clustering Routing Protocol (EEZECR) in Wireless Sensor Network" international journal of Circuits and System, jan2014

[9] Hwa Young Lim, Sung Soo Kim et al "Maximum Energy Routing Protocol based on Strong Head in Wireless Sensor Networks" IEEE International Conference on Advanced Language Processing and Web Information Technology,pp414 - 4192007.

[10] Bilal Abu Bakr, Leszek Lilie, "A Quantitative Comparison of Energy Consumption and WSN Lifetime for LEACH and LEACH-SM " , 31st ICDCS Workshops 2011 ,pp. 182-191, June20-14, 2011.

[11] Chi-Tsun Cheng, Chi K. Tse et al "A Delay-Aware Data Collection Network Structure for Wireless Sensor Networks" Sensors Journal, IEEE, pp. 699 710, March 2011.

[12] M. Nikodem and B. Wojciechowski. "Upper Bounds on Network Lifetime for Clustered Wireless Sensor Networks". NTMS, pages 1-6, 2011.

[13] Yan Shen, Hui Ju "Energy-Efficient Task Assignment Based on Entropy Theory and Particle Swarm Optimization Algorithm for Wireless Sensor Networks".

[14] F.J. Atero, J.J. Vinagre, et al "A Low Energy and Adaptive Architecture for Efficient Routing and Robust Mobility Management in Wireless Sensor Networks"31st International Conference on Distributed Computing Systems Workshops, pp. 172 - 181,20-24 June 2011.

[15] Rongxin Li, Chaomei Zheng, et al "Study of PowerAware Routing Protocol in Wireless Sensor Networks" International Conference on Electrical and Control Engineering (ICECE), pp. 3173 - 3176, 16-18 Sept. 2011.

[16] Jacques M. Bahi, Mohammed Haddad, et al "Distributed Lifetime Optimization in Wireless Sensor Networks"13th International Conference on High Performance Computing and Communications (HPCC), pp. 432 - 439, 2-4 Sept. 2011.

[17] Nagarajan. M, Dr. S. Karthikeyan “ A New Approach to Increase the Life Time and Efficiency of Wireless Sensor Network "International Conference on Pattern Recognition, Informatics and Medical Engineering (PRIME), pp. 231 - 235, 21-23 March 2012.

[18] Young Sang Yun, Ye Xia, et al "Distributed Algorithm for Lifetime Maximization in DelayTolerant Wireless Sensor Network with Mobile Sink" 49th IEEE Conference on Decision and Control (CDC), pp. 370 - 375, 15-17 Dec. 2010.

[19] Jia Xu ,Ning Jin, et al "Improvement of $L E A C H$ protocol for WSN"9th International Conference on Fuzzy Systems and Knowledge Discovery (FSKD), pp. $2174-2177,29-31$ May 2012. 